1

98. Jahrgang

Januar 2021, S. 17 - 20

ISSN 0932-8351

Sonderdruck

\section{Bautechnik}

Zeitschrift für den gesamten Ingenieurbau

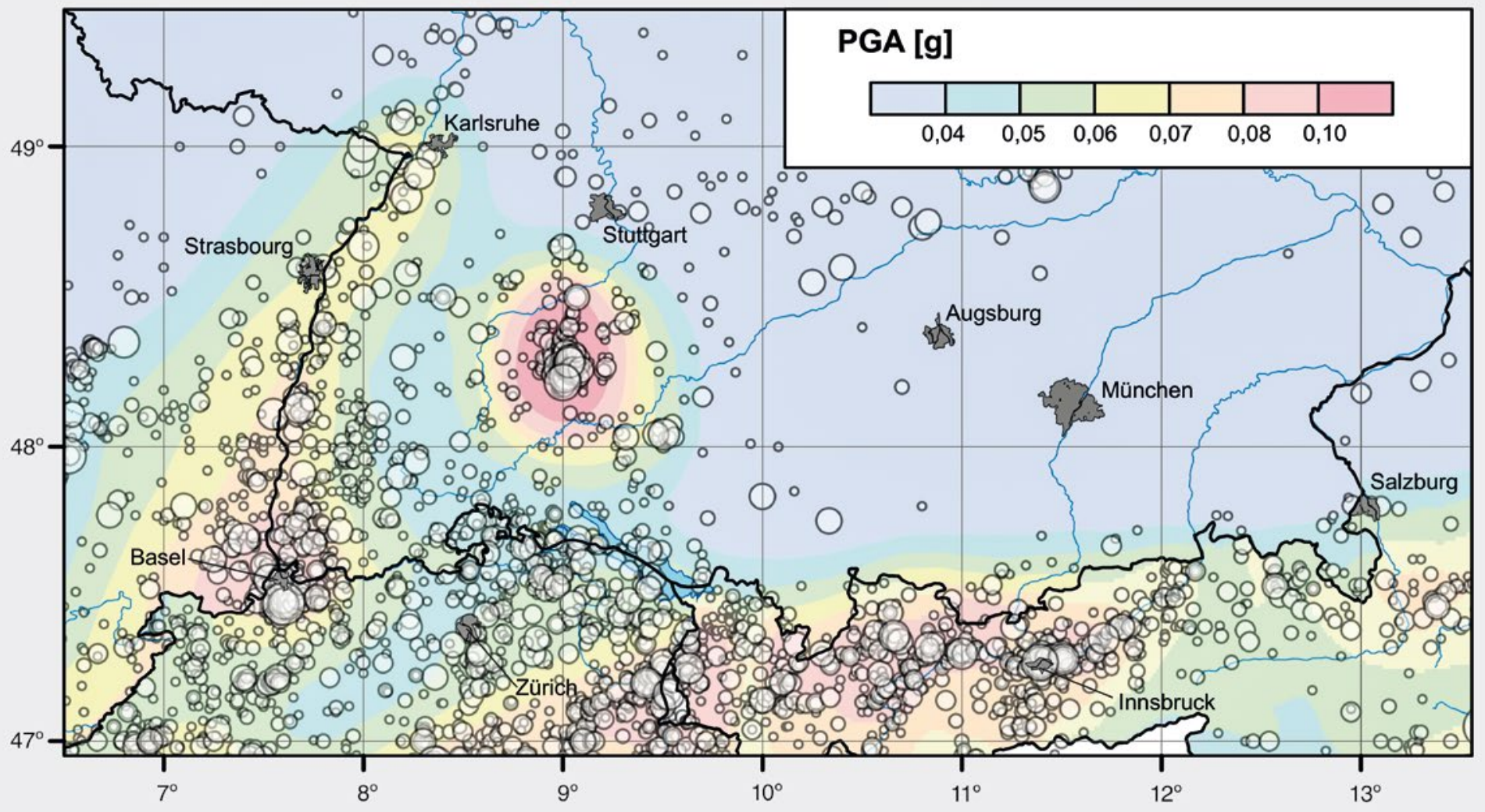

\title{
Vergleich der neuen Erdbebengefährdungs- karten der Schweiz, Deutschlands, Österreichs und Frankreichs für die Nationalen Anhänge zum Eurocode 8
}

Gottfried Grünthal

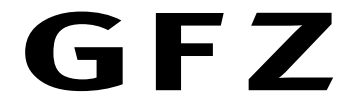





\section{Vergleich der neuen Erdbebengefährdungskarten der Schweiz, Deutschlands, Österreichs und Frankreichs für die Nationalen Anhänge zum Eurocode 8}

Der Vergleich der neuen Erdbebengefährdungskarten für die Schweiz, Deutschland, Österreich und für Frankreich, die für die Nationalen Anhänge zum Eurocode 8 entwickelt wurden, ist Gegenstand dieser Studie. Die vorgestellte gemeinsame Karte überdeckt den südlichen Teil Deutschlands samt angrenzender Gebiete der genannten Nachbarländer. Die Parametrisierung der Karte erfolgt für die Spitzenbodenbeschleunigung, für eine mittlere Wiederholungsperiode von 475 Jahren, für Medianwerte und für einen Untergrund mit einer mittleren Scherwellengeschwindigkeit der obersten $30 \mathrm{~m}$ von $800 \mathrm{~m} / \mathrm{s}$. Entlang des Grenzverlaufs zwischen Deutschland und den südlichen Nachbarländern zeigen die Konturlinien der unabhängig voneinander berechneten Erdbebengefährdungskarten insgesamt eine erstaunlich gute Übereinstimmung. Das gilt insbesondere für Gebiete relativ erhöhter Erdbebengefährdung. Diese gute bis sehr gute Übereinstimmung kann als Indiz für die Belastbarkeit der Einschätzungen angesehen werden.

Stichworte Erdbebengefährdungskarten; Nationale Anhänge für EC 8; Kartenvergleich; Deutschland; Frankreich; Schweiz; Österreich

\section{$1 \quad$ Einführung}

Neueinschätzungen der Erdbebengefährdung zur Nutzung für Nationale Anhänge zum Eurocode 8 liegen aus den letzten Jahren für eine Reihe von Ländern vor, so auch für die Schweiz, Deutschland, Österreich und für Frankreich. Gegenstand der Mitteilung ist der Vergleich dieser Karten entlang der gemeinsamen Grenzen dieser Länder.

Grenzüberschreitende Vergleiche berechneter seismischer Lastannahmen sind zum einen relevant für praktische Belange des Erdbebeningenieurwesens und zum anderen im Hinblick auf die Belastbarkeit unabhängig voneinander gewonnener Resultate seismischer Gefährdungsstudien, d.h., inwieweit realistische Bodenbeschleunigungen ermittelt werden konnten. Hierzu gehören Fragen wie z.B. zur erdbebengerechten Konstruktion länderüberschreitender Verkehrswegebauten, Pipelines etc. Grundsätzlich besteht die berechtigte Forderung, dass zu einem europäischen Regelwerk zum erdbebengerechten Konstruktionsentwurf möglichst grenzüberschreitend harmonisierte Karten der Erdbebengefährdung als Bestandteil der Nationalen Anhänge zur Norm gehören sollten.

Der hier vorgenommene Vergleich ist jedoch insbesondere von ingenieurseismologischem Interesse, d.h. im Hin-
Comparison of the new seismic hazard maps of Switzerland, Germany, Austria and France for the National Annexes to Eurocode 8

The comparison of the new seismic hazard maps for Switzerland, Germany, Austria and France, which were developed for the National Annexes to Eurocode 8, is the subject of this study. The presented common map covers the southern part of Germany together with adjacent areas of the mentioned neighbouring countries. The map is parameterised for peak soil acceleration (PGA), for a mean return period of 475 years, for median values and for a subsoil with a mean shear wave velocity of the uppermost $30 \mathrm{~m}$ of $800 \mathrm{~m} / \mathrm{s}$. Along the boundary between Germany and its southern neighbours, the contour lines of the independently calculated seismic hazard maps show an astonishingly good overall agreement. Slightly more significant deviations are only observed for areas with lower seismic hazard. This good to very good agreement can be seen as an indication of the resilience and thus realistic assessments.

Keywords seismic hazard maps; National Annexes for EC 8; map comparison; Germany; France; Switzerland; Austria

blick auf die Zuverlässigkeit der betrachteten seismischen Gefährdungsstudien. So sollten möglichst ähnliche Bodenbeschleunigungen bzw. Antwortbeschleunigungen anhand der unabhängig voneinander vorgenommenen nationalen seismischen Gefährdungsabschätzungen entlang von Grenzverläufen ein zusätzliches Indiz dafür sein, dass realistische Ergebnisse erzielt werden konnten, sofern diese einzelnen Studien in etwa vergleichbare Bearbeitungstiefen erreicht haben. Jedoch sei betont, dass für eine solche Aussage zur Belastbarkeit weitere Belege zur Verifizierung der Resultate vorliegen sollten, wie sie z.B. in Grünthal et al. [1] angeführt worden sind.

Nicht unerwähnt sollen in dem Zusammenhang ökonomische Gesichtspunkte bleiben, die sich aus grob unterschiedlichen Resultaten, d.h. berechneten Bodenbeschleunigungen, im Hinblick auf Vor-bzw. Nachteile von Standorten dies- und jenseits einer Grenze ergeben könnten.

Die Veröffentlichungen, auf die für den Kartenvergleich Bezug genommen wird, sind für die Schweiz der Report von Wiemer et al. [2], für Deutschland die Veröffentlichungen von Grünthal et al. [1, 3], für Österreich die Publikation einer vorläufigen Karte von Weginger et al. [4] sowie für Frankreich die Arbeiten von Drouet et al. 


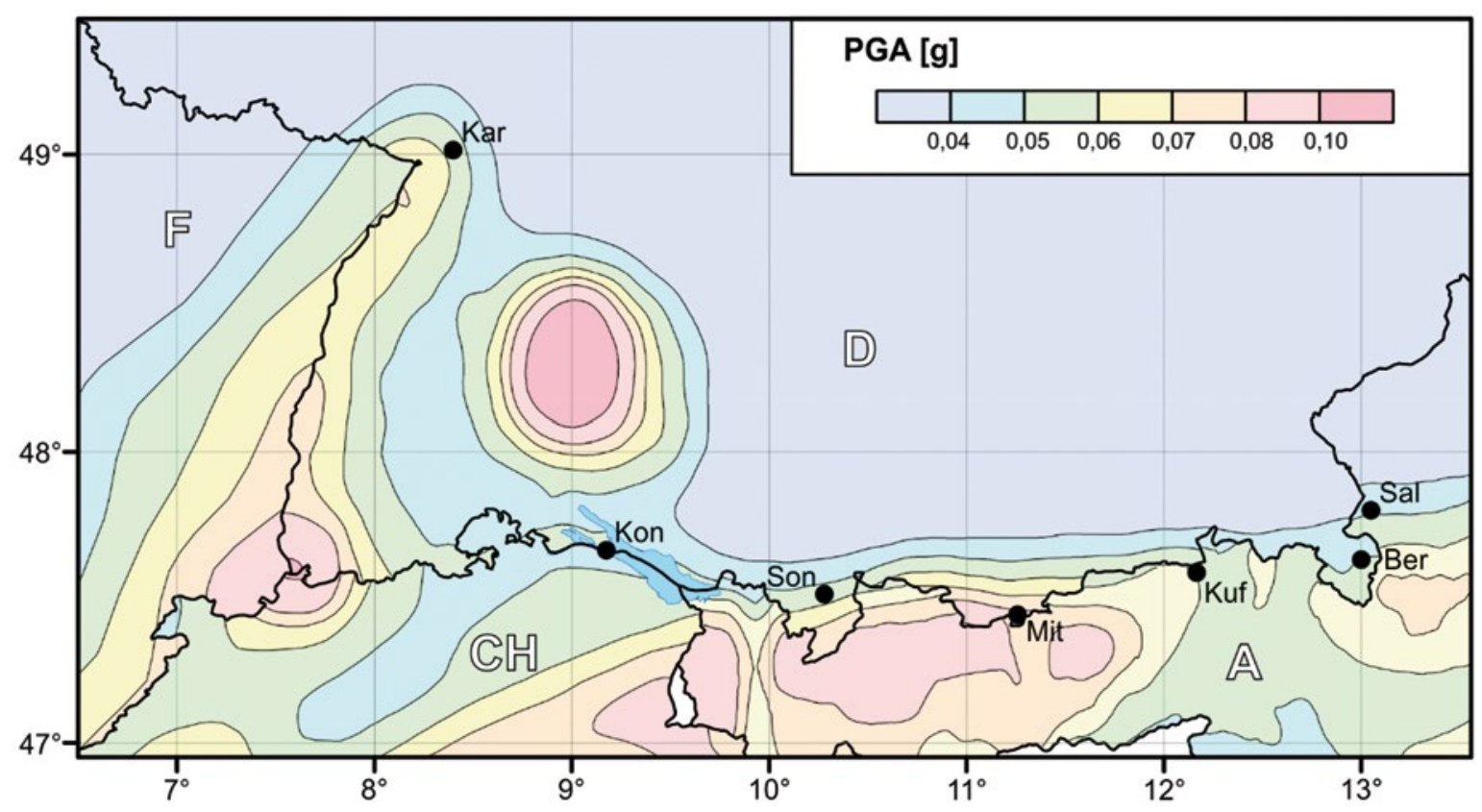

Bild 1 Ausschnitt der Karte der Neueinschätzung der Erdbebengefährdung Deutschlands [1, 3, 7] im Vergleich zu analogen Karten für angrenzende Teile der Schweiz [2], Österreichs [4] und Frankreichs [5,6] - dargestellt sind die Spitzenbodenbeschleunigungen (PGA) in Teilen der Erdbeschleunigung $g$ für eine mittlere Wiederholungsperiode RP von 475 Jahren sowie für Medianwerte und Berechnungen für einen Untergrund mit einer mittleren Scherwellengeschwindigkeit der obersten $30 \mathrm{~m}$ von $v_{S 30}=800 \mathrm{~m} / \mathrm{s}$ (Kürzel der im Text herangezogenen Orte von links nach rechts: Kar - Karlsruhe, Kon - Konstanz, Son - Sonthofen, Mit - Mittenwald, Kuf - Kufstein, Ber - Berchtesgaden, Sal - Salzburg) Section of the map of the reassessment of seismic hazard of Germany $[1,3,7]$ compared to analogous maps for neighbouring parts of Switzerland [2], Austria [4] and France [5, 6] - the peak ground accelerations (PGA) are shown in parts of the acceleration of gravity $g$ for a mean return period RP of 475 years as well as for median values and calculations for a subsoil with a mean shear wave velocity of the uppermost $30 \mathrm{~m}$ of $v_{\mathrm{S} 30}=800 \mathrm{~m} / \mathrm{s}$ (abbreviations of the locations used in the text from left to right: Kar - Karlsruhe, Kon - Konstanz, Son - Sonthofen, Mit - Mittenwald, Kuf - Kufstein, Ber-Berchtesgaden, Sal - Salzburg)

[5, 6]. Diese werden hier in der Reihenfolge ihres Erscheinens aufgeführt und bereits im Titel in der Form benannt.

Die Ergebnisse der probabilistischen seismischen Hazard-Abschätzungen (PSHA) für die vier Länder liegen in teilweise unterschiedlichen Parametrisierungen vor. Der diesbezügliche kleinste gemeinsame Nenner im Hinblick auf die Darstellung einer gemeinsamen Karte bietet die Parameterwahl für die Spitzenbodenbeschleunigung (PGA), für eine mittlere Wiederholungsperiode RP von 475 Jahren, für Medianwerte und Berechnungen für einen Untergrund mit einer mittleren Scherwellengeschwindigkeit der obersten $30 \mathrm{~m}$ von $v_{\mathrm{S} 30}=800 \mathrm{~m} / \mathrm{s}$. Dieser $v_{\mathrm{S} 30^{-}}$ Wert liegt den Berechnungen für Deutschland, Österreich und für Frankreich zugrunde. Der Bericht bezüglich der Schweizer Studie [2] enthält nur eine Kartenabbildung auf der Grundlage von $v_{\mathrm{S} 30}=800 \mathrm{~m} / \mathrm{s}$, und zwar für PGA und Medianwerte. Zur Neueinschätzung der Erdbebengefährdung für Deutschland [1, 3] liegen für alle Karten mit dem zugehörigen Bericht erweiterter Ergebnispräsentationen [7] auch Mediandarstellungen vor. Die PGAKarte für Österreich nach Weginger et al. [4] und diejenigen für Frankreich [5] zeigen gemäß [6] die Mittelwerte. Dank persönlicher Mitteilungen von S. Drouet bzw. von S. Weginger können für die vorliegende Studie die Mediandarstellungen auch für Frankreich und Österreich genutzt werden.

Ein Vergleich der Karten der Neueinschätzung der Erdbebengefährdung Deutschlands und der Schweiz wurde bereits in [1, 3] vorgenommen. Hierfür wurde für die Schweiz eine als final angesehene Karte verwendet (erhalten von S. Wiemer, pers. Mitteilung 2015) und im Kartenvergleich von $[1,3]$ mit dem zugehörigen $\mathrm{Ab}$ schlussbericht [2] zitiert. Diese Karte ist in der Tat Bestandteil des Berichts, allerdings mit einem in Nuancen differierenden Konturenverlauf, insbesondere dem der 0,05-g-Linie.

\section{Vergleich der Erdbebengefährdungskarten}

Den Vergleich der neuen Erdbebengefährdungskarten der Schweiz, Deutschlands, Österreichs und Frankreichs für die Nationalen Anhänge zum Eurocode 8 zeigt Bild 1 für das Gebiet des südlichen Deutschlands mit angrenzenden Teilen der genannten Nachbarländer. Lichtenstein ist ebenso Teil des Kartenausschnitts. Für Lichtenstein liegen dem Autor bisher keine Daten bzw. Informationen vor, ob hier die Berechnungsergebnisse anhand des schweizerischen Erdbebenmodells [2] gelten, sodass dessen Territorium in Bild 1 unberücksichtigt bleibt. Das betrifft auch den Teil Italiens, der südlich des südöstlichsten Teils von Tirol noch im Kartenausschnitt liegt.

Da nur für Deutschland die Beschleunigungen primär in $\mathrm{m} / \mathrm{s}^{2}$ berechnet wurden $[1,3,7]$, sonst aber in Teilen der Erdbeschleunigung g, wird letztere Größe einheitlich für den Kartenvergleich verwendet. Anhand der publizierten Datenlage konnten für die Vergleichskarte die relevanten 
PGA-Konturlinien von $0,04,0,05,0,06,0,07,0,08$ und $0,10 \mathrm{~g}$ dargestellt werden. Da die Daten entlang der Grenzverläufe mit einer sehr feinen Abstufung in hundertstel Teilen von $g$ vorliegen, gestattet dies, den Vergleich in einer hohen Auflösung vornehmen zu können.

Generell zeigt der Vergleich der Karten von Bild 1 eine im Wesentlichen erstaunlich gute bis sehr gute Übereinstimmung. Das betrifft im südlichen Oberrheingraben, d.h. im Grenzgebiet zu Frankreich, die Konturlinien von 0,07 und $0,08 \mathrm{~g}$, welche quasi völlig übereinstimmen. Lediglich die 0,06-g-Konturlinie zeigt westlich von Karlsruhe eine etwas weitere Ausdehnung, als deren Verlauf auf französischer Seite erkennen lässt.

Auch im Dreiländereck von Frankreich, der Schweiz und Deutschland, d.h. im Großraum von Basel, sowie entlang der Grenze zur Schweiz zeigen die Konturen für 0,10, 0,08 und $0,07 \mathrm{~g}$ einen fast identischen Verlauf. Weiter östlich weisen die beiden Konturlinien für noch niedrigere Werte, d.h. für 0,06 und 0,05 g, entlang des Hochrheins in Deutschland etwas höhere Werte auf. Absolut sind diese Unterschiede jedoch minimal. So werden z.B. im Raum Konstanz die Werte von 0,05 g nur knapp überschritten und unmittelbar südlich davon in der Schweiz knapp unterschritten.

Auch im Grenzgebiet zu Österreich ist die Übereinstimmung auffällig gut. Die Karten sind z. B. bezüglich der 0,05- bis 0,07-g-Konturen in den östlichen Allgäuer Alpen im Raum Sonthofen oder in den östlichen Bayerischen Voralpen bzw. den westlichen Chiemgauer Alpen (vgl. die 0,05-g-Kontur unmittelbar nördlich Kufstein) de facto identisch. Im Gebiet der westlichen Allgäuer Alpen zeigt die österreichische Karte um bis zu max. 1/100 g höhere Werte. Im Bereich der Ammergauer Alpen und der westlichen Bayerischen Voralpen zeigt die österreichische Karte dagegen um $<1 / 100 \mathrm{~g}$ kleinere PGA. Im Areal des Wettersteingebirges weisen beide Karten $0,08 g$ auf. Für die zentralen und östlichen Chiemgauer Alpen als auch für die Berchtesgadener Alpen wurden für die deutsche Karte geringere PGA-Werte berechnet als für die umliegenden Gebiete in Österreich einschließlich des Raums Salzburg.

\section{Literatur}

[1] Grünthal, G.; Stromeyer, D.; Bosse, C.; Cotton, F.; Bindi, D. (2018) The probabilistic seismic hazard assessment of Germany - version 2016, considering the range of epistemic uncertainties and aleatory variability in: Bulletin of Earthquake Engineering 16, no. 10, pp. 4339-4395. https://doi. org/10.1007/s10518-018-0315-y

[2] Wiemer, S.; Danciu, L.; Edwards, B.; Marti, M.; Fäh, D.; Hiemer, S.; Wössner, J.; Cauzzi, C.; Kästli, P.; Kremer, K. (2016) Seismic Hazard Model 2015 for Switzerland (SUIhaz2015). Technical Report. Swiss Seismological Service (SED) at ETH Zurich. 10.12686/a2

[3] Grünthal, G.; Stromeyer, D.; Bosse, C.; Cotton, F.; Bindi, D. (2018) Neueinschätzung der Erdbebengefährdung Deutsch-
Eine Höherbewertung trifft auch für den westlichsten Teil vom österreichischen Vorarlberg im Vergleich zur angrenzenden Schweiz zu. Gleiches wird weiter westlich im Grenzgebiet zwischen der Schweiz und Frankreich im Gebiet vom Elsgau und dem Neuenburgischen Jura beobachtet, d.h. kleinere Werte in der Schweiz als im benachbarten Frankreich. Das bedeutet, dass die Bewertungen für die Schweiz in großen Teilen etwas geringer ausfallen als in den beschriebenen benachbarten Gebieten. Betont sei hier nochmals, dass es sich um Unterschiede in einer sehr feinen Abstufung von Konturlinien von 1/100 $g$ handelt, d.h., bez. der Absolutwerte sind die beschriebenen Differenzen marginal.

\section{3}

\section{Schlussfolgerungen}

Der vorgestellte Vergleich der Median-PGA-Karten anhand der Neueinschätzungen der Erdbebengefährdung für Deutschland und denen der südlichen Nachbarländer Frankreich, Schweiz und Österreich zeigt generell eine überraschend gute Übereinstimmung. Die ausgesprochen große Ähnlichkeit wird insbesondere in Arealen relativ erhöhter Erdbebengefährdung beobachtet. Dieser Befund ist ein deutliches Indiz dafür, dass mit den hier betrachteten und unabhängig voneinander vorgenommenen Studien zur Einschätzung der seismischen Gefährdung offensichtlich belastbare und realistische Resultate vorliegen. Zumindest dürfte diese Aussage für das hier betrachtete Untersuchungsgebiet, d.h. den Grenzbereich zwischen Deutschland und seinen südlichen Nachbarländern, zutreffen. Andererseits kann konstatiert werden, dass die vorliegenden Erdbebengefährdungskarten einen hohen Grad einer grenzüberschreitenden Harmonisierung repräsentieren und damit ingenieurseismologische Grundlagen für gut abgestimmte Nationalen Anhänge zur Norm vorliegen.

\section{Dank}

Ein besonderer Dank gilt Stéphane Drouet und Stefan Weginger für die Bereitstellung der im Aufsatz beschriebenen digitalen Daten. lands - Version 2016 - für DIN EN 1998-1/NA in: Bautechnik 95, H. 5, S. 371-384. https://doi.org/10.1002/ bate. 201700098

[4] Weginger, S.; Jia, Y.; Papi-Isaba, M.; Lenhardt, W.; Hausmann, H. (2019) Entwicklung einer regionalen Erdbebengefährdungskarte für Österreich in: Adam, C.; Achs, G.; Furtmüller, T. [Hrsg.] Tagungsband der 16. D-A-CH Tagung Erdbebeningenieurwesen \& Baudynamik. Innsbruck, 26./27. September 2019. S. 27-34.

[5] Drouet, S.; Ameri, G.; Le Dortz, K.; Secanell, R. (2020) A probabilistic seismic hazard map for the metropolitan France in: Bulletin of Earthquake Engineering 18, pp. 18651898. https://doi.org/10.1007/s10518-020-00790-7 
[6] Drouet, S.; Ameri, G.; Le Dortz, K.; Secanell, R. (2020) Correction to: A probabilistic seismic hazard map for the metropolitan France in: Bulletin of Earthquake Engineering. [online first publication]. https://doi.org/10.1007/s10518020-00889-x

\author{
Autor \\ Prof. Dr. Gottfried Grünthal (Korrespondenzautor) \\ ggrue@gfz-potsdam.de \\ ehem. Helmholtz-Zentrum Potsdam \\ Deutsches GeoForschungsZentrum GFZ \\ Sektion 2.6 Erdbebengefährdung und dynamische Risiken \\ Telegrafenberg \\ 14473 Potsdam
}

[7] Grünthal, G.; Stromeyer, D.; Bosse, C. (2017) The data sets of the earthquake model for the probabilistic seismic hazard assessment of Germany, version 2016 - Report on supplementary material for the respective publication. Scientific Technical Report STR 17/05. GeoForschungsZentrum Potsdam. https://doi.org/10.2312/GFZ.b103-17056

\section{Zitieren Sie diesen Beitrag}

Grünthal, G. (2021) Vergleich der neuen Erdbebengefährdungskarten der Schweiz, Deutschlands, Österreichs und Frankreichs für die Nationalen Anhänge zum Eurocode 8. Bautechnik 98, H. 1, S. 17-20. https://doi.org/10.1002/bate.202000052

Dieser Aufsatz wurde in einem Peer-Review-Verfahren begutachtet. Eingereicht: 4. Juni 2020; angenommen: 23. Juli 2020. 



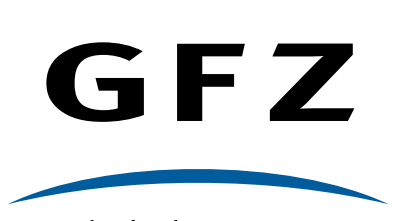

Helmholtz-Zentrum POTS D M

GFZ GeoForschungszentrum Helmholtz-Zentrum Potsdam Telegrafenberg C3

14473 Potsdam

Tel. +49(0)331 288-0 info@gfz-potsdam.de www.gfz-potsdam.de 